\title{
On A Multiple Hilbert's Type Integral Inequality with Non-homogeneous Kernel
}

\author{
Baoju Sun \\ Department of Mathematics \\ Zhejiang Water Conservancy \& Hydropower University \\ Hangzhou, Zhejiang 310018, China \\ Email address: sunbj@zjwchc.com
}

\begin{abstract}
By introducing the norm $\|x\|$ and two parameters $\alpha, \beta$, a multiple Hilbert's type integral inequality with a nonhomogeneous kernel and a best possible constant factor is given.
\end{abstract}

Keywords-Inequalities; Hilbert's inequality; multiple Hilbert's integral inequality; best possible constant

\section{INTRODUCTION}

The well known Hardy-Hilbert's integral inequality is given by (see [2, 8])

$$
\begin{aligned}
& \int_{0}^{\infty} \int_{0}^{\infty} \frac{f(x) g(y)}{x+y} d x d y \\
& <\frac{\pi}{\sin (\pi / p)}\left[\int_{0}^{\infty} f^{p}(t) d t\right]^{1 / p}\left[\int_{0}^{\infty} g^{q}(t) d t\right]^{1 / q},
\end{aligned}
$$

where the constant factor $\pi / \sin (\pi / p)$ is the best possible.

Hardy-Hilbert's integral inequality is inequality with homogeneous kernel, it is important in analysis and applications. During the past few years, many researchers obtained various generalizations, variants and extensions of the inequality of (1.1) (see [1, 6, 7, 9-12] and the references cited therein).

Recently, Yang gave a inequality with non-homogeneous kernel (see [15]).

If $p>1,1 / p+1 / q=1,0<\alpha<\gamma, \beta>-1, f, g \geq 0$, satisfy

$0<\int_{0}^{\infty} t^{p(1+\alpha)-1} f^{p}(t) d t<\infty, 0<\int_{0}^{\infty} t^{q(1+\alpha)-1} g^{q}(t) d t<\infty$, then one has

$$
\begin{aligned}
& \int_{0}^{\infty} \int_{0}^{\infty}(\min \{1 . x y\})^{\gamma}|\ln (x y)|^{\beta} f(x) g(y) d x d y \\
& <\left[\frac{1}{(\gamma-\alpha)^{\beta+1}}+\frac{1}{\alpha^{\beta+1}}\right] \Gamma(\beta+1) \\
& \times\left[\int_{0}^{\infty} t^{p(1+\alpha)-1} f^{p}(t) d t\right]^{1 / p}\left[\int_{0}^{\infty} t^{q(1+\alpha)-1} g^{q}(t) d t\right]^{1 / q},
\end{aligned}
$$

where the constant factor $\left[\frac{1}{(\gamma-\alpha)^{\beta+1}}+\frac{1}{\alpha^{\beta+1}}\right] \Gamma(\beta+1)$ is the best possible.

At present, because of the requirement of higherdimensional harmonic analysis and higher-dimensional operator theory, multiple Hilbert's type integral inequalities have been studied. Y. Hong, B. Yang and J. Kuang etc. obtained some multiple Hilbert's type integral inequalities (see $[3,4,7,13,14])$.

The main objective of this paper is to build multiple Hilbert's type integral inequalities with a non-homogeneous kernel and a best constant factor of (1.2). For this reason, we introduce signs as

$\mathbb{R}_{+}^{n}=\left\{x=\left(x_{1}, x_{2}, \cdots, x_{n}\right): x_{1}, x_{2}, \cdots, x_{n}>0\right\}$,

$\|x\|=\left(x_{1}^{2}+x_{2}^{2}+\cdots+x_{n}^{2}\right)^{\frac{1}{2}}$, and we agree on $\|x\|<c$ representing $\left\{x \in \mathbb{R}_{+}^{n}:\|x\|<c\right\}$.

\section{LEMMAS}

Lemma 2.1.([14]) If $p>0, \alpha>0, f(\tau)$ is a measurable function, then

$$
\begin{gathered}
\int_{x_{1}, x_{2}, \cdots, x_{n}>0 ; x_{1}^{\alpha}+x_{2}^{\alpha}+\cdots+x_{n}^{\alpha} \leq 1} f\left(x_{1}^{\alpha}+x_{2}^{\alpha}+\cdots+x_{n}^{\alpha}\right) d x_{1} d x_{2} \cdots d x_{n} \\
=\frac{\Gamma^{n}\left(\frac{1}{\alpha}\right)}{\alpha^{n} \Gamma\left(\frac{n}{\alpha}\right)} \int_{0}^{1} f(\tau) \tau^{\frac{n}{\alpha}-1} d \tau \\
\int_{x_{1}, x_{2}, \cdots, x_{n}>0 ; x_{1}^{\alpha}+x_{2}^{\alpha}+\cdots+x_{n}^{\alpha} \geq 1} f\left(x_{1}^{\alpha}+x_{2}^{\alpha}+\cdots+x_{n}^{\alpha}\right) d x_{1} d x_{2} \cdots d x_{n} \\
=\frac{\Gamma^{n}\left(\frac{1}{\alpha}\right)}{\alpha^{n} \Gamma\left(\frac{n}{\alpha}\right)} \int_{1}^{\infty} f(\tau) \tau^{\frac{n}{\alpha}-1} d \tau
\end{gathered}
$$


Lemma 2.2. If $n-1<\alpha<n-1+\gamma, \beta>-1$, Define the weight function $w(y)$ as:

$w(y)=\int_{\mathbb{R}_{+}^{n}}(\min \{1,\|x\|\|y\|\})^{\gamma}|\ln (\|x\|\|y\|)|^{\beta}\|x\|^{-1-\alpha} d . x$ Then

$$
\begin{aligned}
& w(y)=\|y\|^{\alpha-n+1} \frac{\Gamma^{n}\left(\frac{1}{2}\right)}{2^{n-1} \Gamma\left(\frac{n}{2}\right)} \\
& \times\left[\frac{1}{(\gamma+n-1-\alpha)^{\beta+1}}+\frac{1}{(\alpha-n+1)^{\beta+1}}\right] \Gamma(\beta+1) .
\end{aligned}
$$

Proof. By (2.1), (2.2),

$$
w(y)=\frac{\Gamma^{n}\left(\frac{1}{2}\right)}{2^{n} \Gamma\left(\frac{n}{2}\right)}
$$

$\times \int_{0}^{\infty}\left(\min \left\{1,\|y\| t^{\frac{1}{2}}\right\}\right)^{\gamma}\left|\ln \left(\|y\| t^{\frac{1}{2}}\right)\right|^{\beta} t^{-\frac{1+\alpha}{2}} t^{\frac{n}{2}-1} d t$

$=\frac{\Gamma^{n}\left(\frac{1}{2}\right)}{2^{n} \Gamma\left(\frac{n}{2}\right)} 2\|y\|^{\alpha-n+1} \int_{0}^{\infty}(\min \{1, u\})^{\gamma}|\ln u|^{\beta} u^{n-\alpha-2} d u$

$=\frac{\Gamma^{n}\left(\frac{1}{2}\right)}{2^{n-1} \Gamma\left(\frac{n}{2}\right)}\|y\|^{\alpha-n+1} \int_{0}^{1}(-\ln u)^{\beta} u^{n-\alpha-2+\gamma} d u$

$+\frac{\Gamma^{n}\left(\frac{1}{2}\right)}{2^{n-1} \Gamma\left(\frac{n}{2}\right)}\|y\|^{\alpha-n+1} \int_{1}^{\infty}(\ln u)^{\beta} u^{n-\alpha-2} d u$.

Notice that

$\int_{0}^{1}(-\ln u)^{\beta} u^{n-\alpha-2+\gamma} d u=\int_{0}^{\infty} t^{\beta} e^{-(n-\alpha-1+\gamma) t} d t$

$=\frac{1}{(\gamma+n-1-\alpha)^{\beta+1}} \Gamma(\beta+1)$,

$\int_{1}^{\infty}(\ln u)^{\beta} u^{n-\alpha-2} d u=\int_{0}^{\infty} t^{\beta} e^{(n-\alpha-1) t} d t$

$=\frac{1}{(\alpha-n+1)^{\beta+1}} \Gamma(\beta+1)$.

The lemma 2.2 is proved.
Lemma 2.3. If $p>1,1 / p+1 / q=1$,

$n-1<\alpha<n-1+\gamma, \beta>-1, n \in \mathbb{Z}, \varepsilon>0$, then $\tilde{I}=\int_{\|x\| \leq 1} \int_{\|y\|>1}(\min \{1,\|x\|\|y\|\})^{\gamma}|\ln (\|x\|\|y\|)|^{\beta}$

$\|x\|^{-\alpha+\frac{\varepsilon}{p}-1}\|y\|^{-\alpha-\frac{\varepsilon}{q}-1} d x d y$

$$
=\left[\frac{\Gamma^{n}\left(\frac{1}{2}\right)}{2^{n-1} \Gamma\left(\frac{n}{2}\right)}\right]^{2} \Gamma(\beta+1)
$$

$\times\left[\frac{1}{(\gamma+n-1-\alpha)^{\beta+1}}+\frac{1}{(\alpha-n+1)^{\beta+1}}\right] \frac{1}{\varepsilon}(1+o(1))$.

$$
\varepsilon \rightarrow 0^{+} \text {.(2.5) }
$$

Proof. By (2.1), (2.2),

$\tilde{I}=\int_{\|y\|>1}\|y\|^{-\alpha-\frac{\varepsilon}{q}-1}$

$\times\left(\int_{\|x\| \leq 1}(\min \{1,\|x\|\|y\|\})^{\gamma}|\ln (\|x\|\|y\|)|^{\beta}\|x\|^{-\alpha+\frac{\varepsilon}{p}-1} d x\right) d y$ $=\int_{\|y\|>1}\|y\|^{-\alpha-\frac{\varepsilon}{q}-1}$

$\left(\frac{\Gamma^{n}\left(\frac{1}{2}\right)}{2^{n} \Gamma\left(\frac{n}{2}\right)} \int_{0}^{1}\left(\min \left\{1,\|y\| t^{\frac{1}{2}}\right\}\right)^{\gamma}\left|\ln \left(\|y\| t^{\frac{1}{2}}\right)\right|^{\beta} t^{\frac{1}{2}\left(n-\alpha+\frac{\varepsilon}{p}-1\right)-1} d t\right) d y$

$=\int_{\|y\|>1}\|y\|^{-n-\varepsilon}\left(\frac{\Gamma^{n}\left(\frac{1}{2}\right)}{2^{n-1} \Gamma\left(\frac{n}{2}\right)} \int_{0}^{\|y\|}(\min \{1, u\})^{\gamma}|\ln u|^{\beta} t^{n-2-\alpha+\frac{\varepsilon}{p}} d u\right) d y$

$=\int_{\| y \mid>1}\|y\|^{-n-\varepsilon}\left(\frac{\Gamma^{n}\left(\frac{1}{2}\right)}{2^{n-1} \Gamma\left(\frac{n}{2}\right)} \int_{0}^{1}(-\ln u)^{\beta} u^{n-\alpha-2+\gamma+\frac{\varepsilon}{p}} d u\right) d y$

$=\int_{\|y\|>1}\|y\|^{-n-\varepsilon}\left(\frac{\Gamma^{n}\left(\frac{1}{2}\right)}{2^{n-1} \Gamma\left(\frac{n}{2}\right)} \int_{1}^{\|y\|}(\ln u)^{\beta} u^{n-\alpha-2+\frac{\varepsilon}{p}} d u\right) d y$ 
$=\left(\frac{\Gamma^{n}\left(\frac{1}{2}\right)}{2^{n-1} \Gamma\left(\frac{n}{2}\right)}\right)^{2} \frac{1}{\varepsilon} \int_{0}^{1}(-\ln u)^{\beta} u^{n-\alpha-2+\gamma+\frac{\varepsilon}{p}} d u$
$+\frac{1}{2}\left(\frac{\Gamma^{n}\left(\frac{1}{2}\right)}{2^{n-1} \Gamma\left(\frac{n}{2}\right)}\right)^{2} \int_{1}^{\infty} t^{\frac{-\varepsilon}{2}-1}\left(\int_{1}^{t^{\frac{1}{2}}}(\ln u)^{\beta} u^{n-2-\alpha+\frac{\varepsilon}{p}} d u\right) d t$

Notice that

$\int_{0}^{1}(-\ln u)^{\beta} u^{n-\alpha-2+\gamma+\frac{\varepsilon}{p}} d u$

$=\frac{1}{\left(\gamma+n-1-\alpha+\frac{\varepsilon}{p}\right)^{\beta+1}} \Gamma(\beta+1)$,

$\int_{1}^{\infty} t^{\frac{-\varepsilon}{2}-1}\left(\int_{1}^{t^{\frac{1}{2}}}(\ln u)^{\beta} u^{n-2-\alpha+\frac{\varepsilon}{p}} d u\right) d t$

$=2 \int_{1}^{\infty} s^{-\varepsilon-1}\left(\int_{1}^{s}(\ln u)^{\beta} u^{n-2-\alpha+\frac{\varepsilon}{p}} d u\right) d s$

$=2 \int_{1}^{\infty}\left(\int_{u}^{\infty} s^{-\varepsilon-1} d s\right)(\ln u)^{\beta} u^{n-2+\alpha+\frac{\varepsilon}{p}} d u$

$=\frac{2}{\varepsilon} \int_{1}^{\infty}(\ln u)^{\beta} u^{n-2-\alpha-\frac{\varepsilon}{q}} d u$

$=\frac{2}{\varepsilon} \frac{1}{\left(\alpha-n+1+\frac{\varepsilon}{q}\right)^{\beta+1}} \Gamma(\beta+1)$.

We have (2.5). The lemma 2.3 is proved.

\section{MAIN RESULTS}

Theorem 3.1. If $p>1,1 / p+1 / q=1, n \in \mathbb{Z}$, $n-1<\alpha<n-1+\gamma, \beta>-1, f, g \geq 0$, satisfy $0<\int_{\mathbb{R}_{+}^{n}}\|x\|^{(1+\alpha) p-n} f^{p}(x) d x<\infty$,

$0<\int_{\mathbb{R}_{+}^{n}}\|y\|^{(1+\alpha) q-n} g^{q}(y) d y<\infty$.

Then

$$
\begin{aligned}
& I:=\int_{\mathbb{R}_{+}^{n}} \int_{\mathbb{R}_{+}^{n}}(\min \{1,\|x\|\|y\|\})^{\gamma}|\ln (\|x\|\|y\|)|^{\beta} \\
& \times f(x) g(y) d x d y
\end{aligned}
$$

$$
\begin{aligned}
& <C_{n}(\alpha, \beta, \gamma)\left[\int_{\mathbb{R}_{+}^{n}}\|x\|^{(1+\alpha) p-n} f^{p}(x) d x\right]^{\frac{1}{p}} \\
& \times\left[\int_{\mathbb{R}_{+}^{n}}\|y\|^{(1+\alpha) q-n} g^{q}(y) d y\right]^{\frac{1}{q}}
\end{aligned}
$$

Where the constant factor

$$
\begin{aligned}
& C_{n}(\alpha, \beta, \gamma)=\frac{\Gamma^{n}\left(\frac{1}{2}\right)}{2^{n-1} \Gamma\left(\frac{n}{2}\right)} \\
& \times\left[\frac{1}{(\gamma+n-1-\alpha)^{\beta+1}}+\frac{1}{(\alpha-n+1)^{\beta+1}}\right] \Gamma(\beta+1) .
\end{aligned}
$$

is the best possible.

Proof. By Hölder 's inequality, one has

$$
\begin{aligned}
& I=\int_{\mathbb{R}_{+}^{n}} \int_{\mathbb{R}_{+}^{n}}(\min \{1,\|x\|\|y\|\})^{\gamma}|\ln (\|x\|\|y\|)|^{\beta} \frac{\|x\| \|^{1+\alpha}}{\|y\|^{\frac{1+\alpha}{p}}} f(x) \\
& \times \frac{\|y\| \frac{1+\alpha}{p}}{\|x\|^{\frac{1+\alpha}{q}}} g(y) d x d y \\
& \leq\left\{\int_{\mathbb{R}_{+}} \int_{\mathbb{R}_{+}^{n}}(\min \{1,\|x\|\|y\|\})^{\gamma} \mid \ln \left(\left.\left.\|x\|\|y\|\right|^{\beta}\right|^{\beta} \frac{\|x\|^{(p-1)(1+\alpha)} f^{p}(x)}{\|y\|^{1+\alpha}} d x d y\right\}^{\frac{1}{p}}\right. \\
& \times\left\{\int_{\mathbb{R}_{+}^{n}} \int_{\mathbb{R}_{+}^{n}}(\min \{1,\|x\|\|y\|\})^{\gamma}|\ln (\|x\|\|y\| \mid)|^{\beta} \frac{\|y\|^{(q-1)(1+\alpha)} g^{q}(y)}{\|x\|^{1+\alpha}} d x d y\right\}^{\frac{1}{q}} \\
& \left.\quad=\left[\int_{\mathbb{R}_{+}^{n}}\|x\|^{(p-1)(1+\alpha)} w(x)\right) f^{p}(x) d x\right]^{\frac{1}{p}} \\
& \quad \times\left[\int_{\mathbb{R}_{+}^{n}}\|y\|^{(q-1)(1+\alpha)} w(y) g^{q}(y) d y\right]^{\frac{1}{q}} .
\end{aligned}
$$

According to the condition of taking equality in Hölder 's inequality, if this inequality takes the form of an equality, then there exist constants $C_{1}$ and $C_{2}$, such that they are not all zero, and

$C_{1}(\min \{1,\|x\|\|y\|\})^{\gamma}|\ln (\|x\|\|y\|)|^{\beta} \frac{\|x\|^{(p-1)(1+\alpha)}}{\|y\|^{1+\alpha}} f^{p}(x)$
$=C_{2}(\min \{1,\|x\|\|y\|\})^{\gamma}|\ln (\|x\|\|y\|)|^{\beta} \frac{\|y\|^{(q-1)(1+\alpha)}}{\|x\|^{1+\alpha}} g^{q}(y)$, a.e.. in $\mathbb{R}_{+}^{n} \times \mathbb{R}_{+}^{n}$. It following that 
$C_{1}\|x\|^{n}\|x\|^{(1+\alpha) p-n} f^{p}(x)=C_{2}\|y\|^{n}\|y\|^{(1+\alpha) q-n} g^{q}(y)=C$ (constant), a.e.. in $\mathbb{R}_{+}^{n} \times \mathbb{R}_{+}^{n}$, which contradicts $(3,1)$, hence we have

$$
\begin{aligned}
& \left.I<\left[\int_{\mathbb{R}_{+}^{n}}\|x\|^{(p-1)(1+\alpha)} w(x)\right) f^{p}(x) d x\right]^{\frac{1}{p}} \\
& \times\left[\int_{\mathbb{R}_{+}^{n}}\|y\|^{(q-1)(1+\alpha)} w(y) g^{q}(y) d y\right]^{\frac{1}{q}} .
\end{aligned}
$$

By lemma 2.2, we have (3.2).

If the constant factor $C_{n}(\alpha, \beta, \gamma)$ in (3.2) is not the best possible, then exists a positive number $k$ ( with $k<C_{n}(\alpha, \beta, \gamma)$ ), such that(3.2) is still valid if one replaces $C_{n}(\alpha, \beta, \gamma)$ by $k$.

For $\varepsilon>0$, sitting

$f_{\varepsilon}(x)=\left\{\begin{array}{cll}\|x\|^{-\alpha+\frac{\varepsilon}{p}-1}, & \|x\| \leq 1 \\ 0, & \|x\|>1,\end{array} g_{\varepsilon}(y)=\left\{\begin{array}{cc}\|y\|^{-\alpha-\frac{\varepsilon}{q}-1}, & \|y\|>1 \\ 0, & \|y\| \leq 1,\end{array}\right.\right.$

$\int_{\mathbb{R}_{+}^{n}} \int_{\mathbb{R}_{+}^{n}}(\min \{1,\|x\|\|y\|\})^{\gamma}|\ln (\|x\|\|y\|)|^{\beta} f_{\varepsilon}(x) g_{\varepsilon}(y) d x d y$

$<k\left[\int_{\mathbb{R}_{+}^{n}}\|x\|^{p(1+\alpha)-n} f_{\varepsilon}^{p}(x) d x\right]^{\frac{1}{p}}\left[\int_{\mathbb{R}_{+}^{n}}\|y\|^{q(1+\alpha)-n} g_{\varepsilon}^{q}(y) d y\right]^{\frac{1}{q}}$,

by lemma 2.3

$\int_{\mathbb{R}_{+}^{n}} \int_{\mathbb{R}_{+}^{n}}(\min \{1,\|x\|\|y\|\})^{\gamma}|\ln (\|x\|\|\mid y\|)|^{\beta} f_{\varepsilon}(x) g_{\varepsilon}(y) d x d y$

$=\int_{\|x\| \leq 1} \int_{\|y\|>1}(\min \{1,\|x\|\|y\|\})^{\gamma}|\ln (\|x\|\|y\|)|^{\beta}$

$\|x\|^{-\alpha+\frac{\varepsilon}{p}-1}\|y\|^{-\alpha-\frac{\varepsilon}{q}-1} d x d y$

$=\left[\frac{\Gamma^{n}\left(\frac{1}{2}\right)}{2^{n-1} \Gamma\left(\frac{n}{2}\right)}\right]^{2} \Gamma(\beta+1)$

$\times\left[\frac{1}{(\gamma+n-1-\alpha)^{\beta+1}}+\frac{1}{(\alpha-n+1)^{\beta+1}}\right] \frac{1}{\varepsilon}(1+o(1))$.

$\left[\int_{\mathbb{R}_{+}^{n}}\|x\|^{p(1+\alpha)-n} f_{\varepsilon}^{p}(x) d x\right]^{\frac{1}{p}}\left[\int_{\mathbb{R}_{+}^{n}}\|y\|^{q(1+\alpha)-n} g_{\varepsilon}^{q}(y) d y\right]^{\frac{1}{q}}$

$=\frac{\Gamma^{n}\left(\frac{1}{2}\right)}{2^{n-1} \Gamma\left(\frac{n}{2}\right)} \cdot \frac{1}{\varepsilon}$. Hence, we have
$\left[\frac{\Gamma^{n}\left(\frac{1}{2}\right)}{2^{n-1} \Gamma\left(\frac{n}{2}\right)}\right]^{2} \Gamma(\beta+1)$

$\times\left[\frac{1}{(\gamma+n-1-\alpha)^{\beta+1}}+\frac{1}{(\alpha-n+1)^{\beta+1}}\right] \frac{1}{\varepsilon}(1+o(1))$.

$\leq k \cdot \frac{\Gamma^{n}\left(\frac{1}{2}\right)}{2^{n-1} \Gamma\left(\frac{n}{2}\right)} \cdot \frac{1}{\varepsilon}$.For $\varepsilon \rightarrow 0^{+}$, we have

$C_{n}(\alpha, \beta, \gamma) \leq k$. This contradicts the fact that $k<C_{n}(\alpha, \beta, \gamma)$, hence the constant factor in (3.2) is the best possible.

\section{REFERENCE}

[1] M.Gao, B.Yang, On the extended Hilbert's inequality, Proceeding of the American Mathematical Society, vol. 126, no. 3, pp. 751-759, 1998.

[2] G. H. Hardy, J. E. Littlewood and G. Pólya, Inequalities, Gambridge University Press, Gambridge, 1952.

[3] Y. Hong, All-sided generalizations about Hilbert's inequalities, Acta Mathematics Sinica, vol. 44, no.4, 619-626, 2001.(in chinese)

[4] Y. Hong, a multiple Hardy- Hilbert integral inequalities with the best constant factor, Journal of Inequalities in Pure and Applied Mathematics, vol. 7, no. 4, article 139, 2006.

[5] L. Hua, An Introduction to advanced mathematics ( Remaining sections), Science publishers, Beijing,1984.

[6] J. Kuang, L. Debnath, On new generalizations of Hilbert's inequality and their applications, Journal of Mathematical Analysis and Applications, vol.245, no.1, 248-265, 2000

[7] J. Kuang, Applied Inequalities, Shandong Science and Technology Press, Jinan, 2004.

[8] D. S. Mitrinovi, J. E. Pecaric and A. M. Fink, Inequalities Involving Functions and Their Integrals and Derivatives, Kluwer Academic Publishers, Boston, 1991.

[9] B. G Pachpatte, On some inequalities similar to Hilbert's inequality, Journal of Mathematical Analysis and Applications, vol.226, no.1, 166$179,1998$.

[10] B. Yang, L. Debnath, On a new strengthened Hardy-Hilbert's inequality, International Journal of Mathematics and Mathematical Sciences, vol.21, no.1, 403-408,1998.

[11] B. Yang, On a generalization of Hardy Hilbert's Integral inequality with a best value, Chinese Annals of Mathematics. Series A, vol.21, no.4, 401-408, 2000.

[12] B. Yang, On a generalization of a Hilbert's Type Integral inequality and Its Applications, Mathematica, Applicata, vol.16 no.2, 82-86, 2003.

[13] B. Yang, On a multiple Hardy Hilbert's Integral inequality, Chinese Annals of Mathematics. Series A, vol.24, no.6, 743-750, 2003.

[14] B. Sun, A Multiple Hilbert type integral inequality with the best contant factor, Journal of inequalities and Applications, vol. 2007, Article ID 71049, 14 page.

[15] B. Yang, An Extended Hilbert type Integral inequality with a nonhomogeneous kernel, Journal of Jilin University(science edition), vol.48, no.5,719-422,2010. 